\title{
Refractory Primary Vitreoretinal Lymphoma
}

National Cancer Institute

\section{Source}

National Cancer Institute. Refractory Primary Vitreoretinal Lymphoma. NCI Thesaurus.

Code C157069.

Primary vitreoretinal lymphoma that does not respond to treatment. 